\title{
Interventionelle Therapie benigner und maligner Gallenwegstenosen
}

\author{
Gesprächsleiter: H. Neuhaus, Düsseldorf \\ Teilnehmer: H.-J. Brambs, Ulm M. W. Büchler, Bern, Schweiz M. Jung, Mainz \\ P. Neuhaus, Berlin J. F. Riemann, Ludwigshafen
}

\section{Einleitung}

Für die palliative Therapie maligner Gallenwegstenosen stehen heute minimalinvasive endoskopische und radiologische Techniken der Stentimplantation als Alternative zur Bypassoperation zur Verfügung. Diese interventionellen Techniken können darüber hinaus auch zur temporären oder definitiven Behandlung benigner biliärer Strikturen eingesetzt werden. Da nur wenige kontrollierte Studien zum Vergleich der einzelnen Verfahren vorliegen, sollte das Vorgehen im Einzelfall interdisziplinär erörtert werden. Das folgende Expertengespräch verdeutlicht, zu welchen Fragen diesbezüglich Konsens besteht und wann ausführliche Diskussionen zwischen den einzelnen Fachdisziplinen erforderlich erscheinen.

\section{Frage: Gibt es Indikationen für eine präoperative endoskopische oder perkutane Drainage bei mali- gnem Verschlußikterus?}

Riemann: In der Regel kommt der ikterische Patient zunächst zur Diagnostik. In diesem Rahmen werden nicht selten intervenierende Maßnahmen durchgeführt, um beispielsweise Material für histologische und zytologische Untersuchungen aus dem Gallengang zu gewinnen. In solchen Fällen ist eine Drainage auch präoperativ häufig zwingend, da theoretisch mit dem Entstehen einer iatrogenen Cholangitis gerechnet werden muß. Eine gesicherte Indikation liegt aus meiner Sicht dann vor, wenn ein hochgradiger Ikterus (Bilirubin über $20 \mathrm{mg} \%$ ) besteht.

Jung: Eine klare Indikation zur biliären Ableitung ist gegeben, wenn als diagnostische Untersuchung eine endoskopische retrograde Cholangiopankreatikographie (ERCP) bzw. perkutane transhepatische Cholangiographie (PTC) mit Einbringen von Kontrastmittel oberhalb der Gallenwegstenose vorgenommen wurde. Da die Kontrastmittelinjektion in gestaute Galle ohne Ableitung eine bakterielle Infektion zur Folge hat, muß während des endoskopischen Eingriffs der stenotische Anteil prothetisch versorgt werden. Überdies ist ein Teil der gestauten Galle bereits primär bakteriell kontaminiert.

\section{KARGER}

Fax +497614520714

E-mail kargergmbh@aol.com

www.karger.com
(C) 1999 S. Karger GmbH, Freiburg

Accessible online at:

http://BioMedNet.com/karger
Grundsätzlich kann auch ohne eine ERCP oder PTC operiert werden, wenn die bildgebenden Verfahren (CT, MRCP etc.) eine ausreichende Diagnostik bieten. Die Notwendigkeit einer generellen präoperativen Ableitung gestauter Gallenwege bei Verschlußikterus ist umstritten. Es existieren aber Daten, die auf einen besseren postoperativen Verlauf nach präoperativer biliärer Drainage hinweisen.

Neuhaus: Ja, aber wahrscheinlich muß nach der Lokalisation und dem voraussichtlichen Operationsverfahren differenziert werden. Bei den zentralen Gallenwegkarzinomen sollten möglichst alle Gallenwegsegmente präoperativ drainiert werden, um schweren postoperativen Komplikationen, insbesondere nach Gallenwegresektion mit Leberteilresektion, aus dem Wege zu gehen. Leberinsuffizienz und Cholangitis treten nach eigener Erfahrung, aber auch nach Meinung anderer Gruppen, besonders häufig auf, wenn bei komplettem Verschlußikterus oder nur partieller Drainage operiert wird.

Beim distalen Gallenwegkarzinom wiederum ist die Frage unentschieden. Die meisten Patienten, die wir operierten, haben eine endoskopische Sphinkterotomie und Stenteinlage erhalten. Beim Bilirubinwert unter $10 \mathrm{mg} / \mathrm{dl}$ operieren wir auch gelegentlich ohne vorherige Drainage.

Büchler: Wir bevorzugen bei allen Patienten, welche ein Bilirubin über $10 \mathrm{mg} \%$ aufweisen, eine präoperative endoskopische Gallenwegdrainge mit einem Plastikstent. Wir sehen durch diese Maßnahme keine Verschlechterung der operativen Gegebenheiten, sondern durch den Rückgang des Ikterus und die dadurch bedingte Senkung der portalen Hypertension eine Verbesserung der operativen Bedingungen. Eine präoperative perkutane Drainage versuchen wir in jedem Fall zu vermeiden, da die Komplikationsrate dieses Verfahrens erheblich ist.

Brambs: Als Indikationen für eine präoperative Drainage bei malignem Verschlußikterus sehe ich 1. die eitrige Cholangitis, 2. einen schlechten Allgemeinzustand zur Überbrückung bis zur geplanten Operation. 
2. Frage: In welchen Fällen würden Sie eine palliative Bypassoperation einer endoskopischen biliären Endoprothetik vorziehen?

Riemann: Die endoskopische biliäre Endoprothetik ist im Regelfall der palliativen Bypassoperation überlegen. Eine operative Drainage ist dann sinnvoll, wenn bei einem Patienten nach Probelaparotomie die Inoperabilität festgestellt worden ist. Hier sollte in der gleichen Sitzung eine bilioduodenale Drainage angelegt werden. Dies gilt auch für solche Patienten, bei denen aus Gründen einer Duodenalstenose eine Gastroenterostomie angelegt wird. Hier kann in gleicher Sitzung in den meisten Fällen eine bilioduodenale Drainage angelegt werden.

Jung: 1. Bei distaler maligner Gallenwegstenose und gleichzeitig bestehender Duodenalstenose, so daß während einer Operation 2 Anastomosen vorgenommen werden können - Gastroenterostomie und Hepatikojejunostomie. 2. Wenn sich während einer als kurativ angesetzten Whipple-Operation der Pankreastumor als irresektabel erweist und eine Gallenweganastomose technisch unproblematisch ist.

Neuhaus: Wenn die voraussichtliche Überlebenszeit des Patienten 1 Jahr oder mehr beträgt, kann man eine palliative Bypassoperation sicher in Betracht ziehen. Weiter diskutieren wir eine Bypassoperation, wenn eine endoskopische Entlastung, z.B. aus anatomischen Gründen, unmöglich ist. Hier sollte dann aber die perkutane Route zur Positionierung eines Stents überlegt werden. Eine perkutane Dauerableitung für längere Zeit halte ich dagegen für ungeeignet.

Büchler: Bei Patienten in gutem Allgemeinzustand, mit normaler, altersentsprechender Funktion von Herz, Lunge, Niere und Leber, bei denen die Lebenserwartung geschätzt über 3 Monate beträgt, ziehen wir eine palliative Bypassoperation einer endoskopischen biliären Endoprothetik vor.

Brambs: Bei hochgradiger Duodenalstenose.

\section{Frage: Welche Form der Palliation streben Sie bei einem fortgeschrittenen Pankreaskarzinom mit biliärer Stenose und Duodenalstenose an (konven- tionelle oder laparoskopische Bypässe, Duodenal- stents, perkutane transhepatische Sonden etc.)?}

Riemann: Beim fortgeschrittenen Pankreaskarzinom kommt es auf die zu erwartende Überlebenszeit an, die man nach dem Karnofsky-Index grob abschätzen kann. Liegt ein Karnofsky-Index über 70 vor, so sind laparoskopische Bypässe das Vorgehen der Wahl, da man, wie unter Frage 2 ausgeführt, beides in einer Sitzung durchführen kann. Bei niedrigem Karnofsky-Index würden wir uns für die endoskopischen Verfahren entscheiden. In einigen Fällen gelingt es, bei kurzer zuführender Schlinge auch über die Gastroenterostomie einen Prothesenaustausch vorzunehmen. Hier sind dann perkutane Verfahren respektive chirurgische Bypässe nicht notwendig.

Jung: Bei nicht passierbarer Duodenalstenose, die chirurgische Option - Hepatikojejunostomie und Gastroenterostomie. Transhepatische Ableitung und laparoskopische Gastroenterostomie sind äquivalente Maßnahmen, falls entsprechende Möglichkeiten vor Ort gegeben sind.

Neuhaus: Ich bevorzuge auf jeden Fall als Palliativmaßnahme Stents. Wenn aber bereits wegen Duodenalstenose eine Gastroenterostomie notwendig wird, kann in gleicher Sitzung durchaus eine biliodigestive Anastomose durchgeführt werden. Dies gilt auch für wenige Fälle, bei denen eine Probelaparotomie die Inoperabilität, z. B. Lebermetastasen, ergibt. Hier kann bei offenem Bauch gelegentlich die biliodigestive Anastomose sinnvoll sein.

Büchler: Bei Patienten mit fortgeschrittenem Pankreaskarzinom mit biliärer Stenose und Duodenalstenose streben wir eine offene operative Therapie an im Sinne eines Doppelbypasses (Hepatikojejunostomie plus Gastrojejunostomie).

Brambs: Perkutane transhepatische Drainage.

\section{Frage: Nach welchen Kriterien wählen Sie chirur- gische, endoskopische oder perkutane Drainagever- fahren bei der Palliation hilärer Gallenwegkarzinome (Klatskin-Tumor)? Ist bei bilateralen Stenosen eine einseitige Ableitung ausreichend?}

Riemann: Die hilären Gallenwegkarzinome der Grade II und III sind klassische Domänen des perkutanen transhepatischen $\mathrm{Zu}$ gangs. Hier kann man nicht nur eine exaktere saubere Diagnostik der Tumorinfiltration, sondern auch eine bessere beidseitige Drainage durchführen. Wir streben, wenn immer möglich, eine doppelseitige Drainage an, da sich nicht nur durch eigene Erfahrung, sondern auch in Mitteilungen der Literatur gezeigt hat, daß die einseitige Drainage mit einem deutlich höheren Cholangitisrisiko behaftet ist. Das gilt nicht, wenn bei der Primärdarstellung eine Seite komplett von der Darstellung ausgeschlossen ist. Hier genügt häufig, das kann durch eigene Daten belegt werden, eine einseitige transhepatische oder transpapilläre Drainage. Chirurgische palliative Verfahren in diesen Situationen lassen wir nicht durchführen.

Jung: Explorative Laparotomie bei kleiner Stenose und jungen Patienten zur Frage der diagnostischen Sicherheit (!) und einer möglichen Resektion.

Als Palliation kommt in erster Linie die perkutane transhepatische Versorgung aufgrund des kürzeren Weges und der damit leichteren Tumorpassage in Betracht. Ideal ist die beidseitige transhepatische Versorgung mit je einem Metallstent bei Verlegung beider Hauptäste. In der Regel gelingt die Tumorpassage und Stentüberbrückung leichter transhepatisch als transpapillär. Bei unproblematischer 
Tumorpassage und fortgeschrittenem Karzinom kann auch eine beidseitige endoskopische transpapilläre Ableitung mit 2 Kunststoffprothesen vorgenommen werden. Der endoskopische Zugang bei hilären Stenosen ist wegen der fehlenden Lebertraumatisierung (PTC) immer der komplikationsärmere Weg. Eine einseitige Ableitung ist in der Regel ausreichend, um den Ikterus zu reduzieren. Sie kann aber zur biliären Infektion durch Eindringen von Kontrastmittel in den gestauten Anteil führen. Eine beidseitige Ableitung ist daher immer vorzuziehen.

Neuhaus: Therapie der 1. Wahl ist in unserer Klinik die endoskopische transpapilläre beidseitige Drainage. Eine einseitige Ableitung ist unzureichend, da es regelmäßig zur Infektion der nicht abgeleiteten Seite kommt. Verschließt der Tumor die einzelnen Segmentgallengänge, besteht nach unserer Erfahrung immer eine sehr schlechte Prognose für den Patienten mit wenigen Wochen Überlebenszeit. Die perkutane Drainage wird bei uns nur in Ausnahmefällen angewendet. Chirurgische Drainagen kommen so gut wie gar nicht mehr beim Klatskin-Tumor zur Anwendung.

Büchler: Bei hilären Gallenwegkarzinomen sollte, wann immer möglich, eine operative Exploration erfolgen. Die operative Therapie von hilären Gallenwegkarzinomen ist viel häufiger möglich als früher angenommen. In der Kombination von Gallenwegresektion mit einer partiellen Leberresektion sind ein Großteil auch der komplizierten hilären Gallenwegkarzinome resektabel. Ist eine Resektion nicht möglich, dann streben wir als erste Maßnahme eine endoskopische Drainage an. Diese kann auch einseitig durchgeführt werden, wenn ein Großteil der Leber (in der Regel die rechte) drainierbar ist. Bei palliativer Situation muß keine beidseitige Drainage erzwungen werden. Ist die endoskopische Drainage nicht möglich, meist aus technischen Gründen, erfolgt in unserer Klinik eine perkutane transhepatische Drainage, die in der überwiegenden Zahl der Fälle erfolgreich ist.

Brambs: a) Operation, wenn nach klinischen Gesichtspunkten (Alter, Allgemeinzustand) und aufgrund der Resultate in bildgebenden Verfahren eine Resektion möglich ist; b) endoskopische Drainageverfahren; c) perkutane transhepatische Drainageverfahren, wenn endoskopische Verfahren nicht möglich sind oder nicht gelingen.

Bei endoskopischen Verfahren ist wahrscheinlich in den meisten Fällen eine einseitige Ableitung ausreichend. Bei perkutanen transhepatischen Verfahren muß eine zwei- oder mehrseitige Ableitung erfolgen, wenn die unterschiedlichen Segmente nicht vollständig abgehängt sind. Das hat technische Gründe, da die Implantation von perkutanen transhepatischen Stents in mehrzeitigen Schritten erheblich schwieriger ist als bei einzeitiger Durchführung. Auch bei Atrophie eines Leberlappens (im Ultraschall, CT oder MRT) auf alle Fälle nur einseitige Ableitung.

\section{Frage: Sollten in der endoskopischen Palliation maligner biliärer Stenosen Kunststoffdrainagen rou- tinemäßig oder nur bei Anzeichen von Komplikatio- nen gewechselt werden?}

Riemann: Diese Frage ist völlig offen. Eine kürzlich veröffentlichte amerikanische Untersuchung hat gezeigt, daß der regelmäßige Wechsel von Kunststoffdrainagen zu einer deutlich geringeren Komplikationsrate führt und im Gesamtergebnis den Metallstents vergleichbar ist. Dies muß aber erst noch durch weitere Studien bewiesen werden. Eine solche Studie wird von uns zusammengestellt. Derzeit wechseln wir Kunststoffdrainagen erst bei Anzeichen von Komplikationen.

Jung: Selbstexpandierende Metallendoprothesen besitzen eine deutlich höhere Offenheitsrate (Median 6-8 Monate) als Kunststoffprothesen und sind daher für eine endoskopische Palliation am besten geeignet. Sie sind bis zu 5- bis 7fach teuerer als Kunststoffstents. Plastikprothesen verstopfen deutlich früher als Metallstents durch Sludge, Gallesalze, bakterielle Überwucherungen sowie Nahrungsmittelreste. Die Wahl der Endoprothese korreliert daher auch mit der Tumorgröße und der Prognose des Patienten. Ein endoskopischer Prothesenwechsel erfolgt nur bei Auftreten von erneutem Ikterus oder Infektionszeichen (Schüttelfrost, Fieber, Anstieg der Bilirubin- und Cholestasewerte, dunkler Urin). Ein routinemäßiger Wechsel erfolgt nicht.

Neuhaus: Das Wechseln der möglichst großlumigen Kunststoffdrainagen sollte in regelmäßigen Abständen, etwa alle 8 Wochen, erfolgen.

Büchler: Die Literaturergebnisse zeigen, daß Kunststoffdrainagen nur bei Anzeichen von Komplikationen (Fieber, Ikterus) zu wechseln sind. Der prophylaktische Wechsel dieses Typs endoskopischer Drainagen ist aus meiner Kenntnis nicht sinnvoll.

Brambs: Ich würde einen routinemäßigen Wechsel vorziehen, da Komplikationen wie Cholangitiden für den Patienten belastender und schließlich wahrscheinlich auch teurer sind.

\section{Frage: Bei welchen Patienten mit malignen oder benignen biliären Stenosen bevorzugen Sie Metall- stents gegenüber Kunststoffprothesen?}

Riemann: Die Implantation von Metallstents ist ein ökonomisches Problem, da es in den meisten Abteilungen für diese Technik keine Sonderentgelte gibt. Grundsätzlich implantieren wir Metallstents bei allen Patienten, bei denen primär ein perkutaner transhepatischer Zugang gewählt worden ist. Metallstents werden bei transpapillärem Zugang dann eingesetzt, wenn bei Patienten mit gutem Karnofsky-Index die Kunststoffprothese rasch okkludiert. 
Jung: Metallstents werden aufgrund ihrer längeren Offenheitsrate und geringen Komplikationsneigung grundsätzlich bei malignen Stenosen bevorzugt. Für benigne Stenosen eignen sich Metallendoprothesen nicht, da die offenen Drahtmaschen ein Durchwachsen von Granulationsgewebe bis hin zum Verschluß ermöglichen. Die durchwachsene Prothese kann endoskopisch nicht extrahiert werden. Benigne Stenosen werden zeitlich begrenzt endoprothetisch versorgt. Daher kommen Kunststoffprothesen mit periodischem Wechsel zum Einsatz.

Neuhaus: Wir bevorzugen immer Kunststoffdrainagen, da die Metallstents für oftmals übersehbare Zeiträume zu teuer sind. Außerdem sind sie nicht mehr mobilisierbar, z. B. bei Tumorprogreß. Überwucherungen an den Enden sind ebenfalls problematisch.

Büchler: Benigne Stenosen der Gallenwege sind aus meiner Sicht nicht mit Metallstents anzugehen. Hier ist der vorübergehende Versuch der Drainage mit einem Plastikstent im gegebenen Fall sinnvoll. Wenn dies nicht zum Erfolg führt, sollte immer eine operative Therapie angestrebt werden. Die chirurgische Therapie von gutartigen Gallenwegstenosen ist in erfahrenen Händen mit sehr guten Ergebnissen vergesellschaftet. Bei malignen Stenosen sollte, wenn eine operative Therapie nicht möglich oder nicht sinnvoll erscheint, frühzeitig ein Metallstent eingelegt werden, da die Ergebnisse der Literatur zeigen, daß die Metallprothesen bei malignen Stenosen eine bessere Langzeitfunktion haben.

Brambs: Metallstents würde ich dann vorziehen, wenn prognostisch noch eine relativ gute Lebenserwartung (über 6 Monate) besteht. Bei benignen Stenosen würde ich primär eine passagere Stentung mit Kunststoffprothesen versuchen. Wenn eine dauerhafte Implantation notwendig ist, würde ich in selektierten Fällen kurze Metallstents bevorzugen.

7. Frage: Würden Sie bei einem Patienten mit durchschnittlichem Operationsrisiko bei einer postoperativen biliären Stenose zur Relaparotomie oder zur endoskopischen bzw. perkutanen Dilatation mit/ ohne anschließender Endoprothetik raten?

Riemann: Die Antwort ist abhängig von der Art der postoperativen Stenose. Bei einem Totalverschluß würde ich unbedingt eine Relaparotomie anstreben. Bei einer Stenose, die sehr hilusnahe sitzt, würde ich ebenfalls einen operativen Eingriff bevorzugen. Alle anderen postoperativen Stenosen sind prinzipiell der endoskopischen Prothetik nach Protokoll sehr gut zugänglich. Eigene Erfahrung über mehr als 7 Jahre belegen ein gutes Ergebnis in über $70 \%$ der so behandelten Patienten.

Jung: Die endoprothetische Versorgung einer postoperativen biliären Stenose mit periodischem Wechsel über 1 Jahr zeigt gleiche Erfolgsraten wie die frühzeitige Hepatikojejunostomie. Über $80 \%$ der Patienten profitieren von einer einjährig durchgeführten endo- prothetischen Versorgung mit definitiver Offenhaltung des Gallengangs. Bei gegebener Passage und Dilatation einer postoperativen biliären Stenose ist daher aktuell die endoskopische Prothetik als Therapie der Wahl anzusehen. Die Strategie beinhaltet die Ballondilatation und das Einbringen von 1-2 10-French-Endoprothesen mit periodischem Wechsel in 3monatigem Abstand. Nach einjähriger prothetischer Versorgung wird die endoskopische Therapie beendet. Bei Eintreten einer Rezidivstenose erfolgt die Entscheidung zur Hepatikojejunostomie. Die biliäre Anastomose kann durch eine seitlich zur Bauchwand verlagerte blinde Schlinge modifiziert werden (permanent access Hepaticojejunostomy). Bei Auftreten einer Rezidivstenose ist eine endoskopische Therapie über die Eröffnung dieses intestinalen Stomas möglich.

Neuhaus: Bei einer postoperativen biliären Stenose und benigner Grunderkrankung empfehle ich zunächst für 6-12 Monate Dilatation und Stent. Dies ist aber immer nur als temporäre Maßnahme anzusehen. Wenn die Stenose nach 12 Monaten weiterhin behandlungsbedürftig ist, empfehle ich eine Relaparotomie. Bei maligner Grunderkrankung scheint es mir immer besser zu sein, eine endoskopische bzw. perkutane Maßnahme mit dauerhafter Stenteinlage vorzunehmen.

Büchler: Bei einem Patienten mit «durchschnittlichem» Operationsrisiko würde ich einen ersten Versuch mit endoskopischer Drainage bzw. Dilatation anstreben. Führt dieser Versuch nicht zum Erfolg, ist unbedingt eine operative Therapie anzustreben. Die operative Therapie solcher Stenosen hat eine sehr hohe Erfolgsrate (über $90 \%$ ).

Brambs: Bei durchschnittlichem Operationsrisiko würde ich eher zu einer erneuten Operation raten.

\section{Frage: Welches therapeutische Konzept vertreten Sie bei benignen Stenosen biliodigestiver Anasto- mosen?}

Riemann: Wenn nach einem operativen Eingriff an den Gallenwegen eine Stenosierung eingetreten ist, so bevorzugen wir in erster Linie den perkutanen Weg. Meistens liegen diese Stenosen sehr hilusnahe (Zustand nach Hepatikojejunostomie). Nach Schaffung eines stabilen Traktes durch die Leber mit Anschluß an das Gallengangsystem kann optimal sondiert werden, auch dann, wenn ein subtotaler Verschluß vorliegt. Wir bevorzugen die Einlage von Yamakawa-Prothesen, die wir in dreimonatigem Abstand wechseln. Kommt es nach Beendigung einer meistens über $1 \mathrm{Jahr}$ gehenden Dilatationsbehandlung zu einer Restenose, so würden wir in Abhängigkeit vom Alter des Patienten erneut perkutan behandeln oder dann einen Metallstent implantieren.

Jung: Zunächst bevorzugen wir die perkutane transhepatische Ballondilatation, gegebenenfalls auch die wiederholte Ballondilatation mit kritischer Indikationsstellung zur Implantation einer Me- 
tallendoprothese. Erst danach erfolgt die Aufhebung der narbigen Stenose und Neuanlage einer biliodigestiven Anastomose, eventuell mit seitlichem Stoma.

Neuhaus: Diese Frage ist bereits im Prinzip beantwortet. Die entzündlichen Umbauvorgänge an biliodigestiven Anastomosen ziehen sich über 6-12 Monate hin, so daß auch in diesem Zeitraum eine wiederholte interventionelle Therapie gerechtfertigt ist. Ist sie nach dieser Zeit nicht erfolgreich, würde ich bei benignen Stenosen immer eine erneute chirurgische Maßnahme bevorzugen.

Büchler: In dieser Situation sollte die operative Therapie die erste Wahl sein. Erfahrungsgemäß sind endoskopische Verfahren bei stenosierten, biliodigestiven Anastomosen nicht von Langzeiterfolg gekrönt. Im Gegensatz dazu ist die operative Therapie stenosierter, biliodigestiver Anastomosen in erfahrenen Händen mit einer hohen Erfolgsrate vergesellschaftet. Da die operative Therapie dieser Situation sehr von der Expertise des Chirurgen abhängt, ist dies eine wichtige Voraussetzung für den Therapieerfolg.

Brambs: Bei perkutanem Zugang würde ich hier primär kurze Metallstents implantieren, unter der Voraussetzung, daß diese Stents so implantiert werden, daß noch ausreichend Platz für eine eventuell notwendig werdende biliodigestive Anastomose besteht.

\section{Frage: Wann und wie behandeln Sie eine durch chronische Pankreatitis bedingte Cholestase endo- skopisch?}

Riemann: Nur in Ausnahmefällen. Eigene Erfahrungen haben gezeigt, daß vor allem bei der alkoholbedingten chronischen Pankreatitis die Mitarbeit der Patienten äußerst schlecht ist. Als Folge der meist langen (patientenbedingten) Liegezeit der Prothesen entstehen bei über $40 \%$ dieser Patienten Choledochosteine, die zum Teil sehr schwer zu behandeln sind und immer wieder zu Cholangitiden führen. Wir empfehlen eine endoskopische Drainage nur in Ausnahmefällen, z.B. dann, wenn aufgrund des Allgemeinzustandes oder der absoluten Ablehnung einer Operation ein anderer Eingriff nicht möglich ist. Behandelt werden sollte nur die symptomatische Cholestase.

Jung: Eine chronische Pankreatitis führt durch entzündliche Veränderungen im Bereich des Pankreaskopfes zur Röhrenstenose des distalen Gallengangs. Diese Gallengangverengung kann ohne, mit reiner enzymatischer Cholestase oder mit einem Ikterus einhergehen. Grundsätzlich sind - abhängig vom Entzündungsgrad und von der narbigen kalzifizierenden Pankreasveränderung - die Gallenwegstenosen rückbildungsfähig. Eine Röhrenstenose mit begleitendem Ikterus bei chronischer Pankreatitis wird aktuell mit der Implantation einer Kunststoffprothese und periodischem Wechsel etwa alle 4 Monate behandelt. Der Benefit einer verbesserten (?) Galleableitung bei Röhrenstenose und Cholestase ohne Ikterus ist nicht bewiesen. Die nur mit einer Cholestase einherge- hende Stenose bei chronischer Pankreatitis ist daher keine Indikation zur endoskopischen Prothesenimplantation, zumal Plastikprothesen zur Frühokklusion neigen und damit nur zusätzliche Probleme (Sludgebildung, biliäre Infektionen) hervorrufen.

Neuhaus: Cholestase bei chronischer Pankreatitis ist zunächst die Domäne der endoskopischen Therapie. Wenn aber dauerhaft eine Stentimplantation notwendig ist, ist sicherlich auch die duodenumerhaltende Pankreaskopfresektion mit Dekompression des D. choledochus nach Beger heute die erfolgversprechendste Behandlung.

Büchler: Die durch chronische Pankreatitis verursachte Cholestase sollte unbedingt operativ angegangen werden. Die Ergebnisse des Langzeitstentings bei chronischer Pankreatitis sind enttäuschend, wohingegen die Ergebnisse der chirurgischen Therapie der Choledochusstenose bei chronischer Pankreatitis (duodenumerhaltende Pankreaskopfresektion) im Langzeitverlauf sehr gut sind. Selbst sehr erfahrene interventionelle Gastroenterologen sind in der Zwischenzeit vom Langzeitstenting der Choledochusstenose bei chronischer Pankreatitis wegen der Problematik des regelmäßigen Stentwechsels und der damit vergesellschafteten multiplen Untersuchungen und Komplikationen für den Patienten wieder abgekommen.

Brambs: Bei dreifach erhöhter alkalischer Phosphatase.

\section{Frage: Wie therapieren Sie dominante Stenosen bei primär sklerosierender Cholangitis?}

Riemann: Dominante Choldochusstenosen sind der endoskopischen Dilatationsbehandlung sehr gut zugänglich. Sie erfolgt meistens durch wiederholte Ballondilatation unter Antibiotikaschutz. Führt das nicht zu einem befriedigenden Ergebnis, können auch Kunststoffendoprothesen eingelegt werden. Hier muß allerdings für einen regelmäßigen Wechsel gesorgt werden.

Jung: Endoprothetisch mit Plastikprothesen im periodischen Wechsel, allerdings nur bei gleichzeitig bestehendem Ikterus und Cholestase.

Neuhaus: Durch wiederholte vorsichtige endoskopische Dilatation.

Büchler: Hier kann eine endoskopische Therapie mit Stent/Dilatation angestrebt werden. Grundsätzlich ist die primär sklerosierende Cholangitis eine Indikation zur Lebertransplantation, weswegen das endoskopische Verfahren nur vorübergehenden Charakter haben kann. Die Gefahr der Entwicklung von cholangiolären Karzinomen ist bei primär sklerosierender Cholangitis groß, weswegen vor einer Langzeittherapie mit endoskopischen Methoden zu warnen ist.

Brambs: Mit Ballondilatation. 


\section{Frage: Gibt es bei Klatskin-Tumoren Indikationen für eine Lebertransplantation?}

Riemann: Selbst durch erfahrene Chirurgen sind nur 25\% der Cholangiokarzinome zum Zeitpunkt der Diagnose operabel. Die 5-Jahres-Überlebensrate ist äußerst niedrig. Vor diesem Hintergrund sollte unter der Voraussetzung von genügend Spenderorganen die Lebertransplantation neu bedacht werden.

Jung: Nein.

Neuhaus: Prinzipiell sind die zentralen Gallenwegkarzinome für eine Lebertransplantation ungeeignet. Im Rahmen klinischer Studien muß aber speziell auch dieser Tumor mit seinen im Prinzip außerordentlich schlechten Heilungsaussichten für innovative
Techniken in Betracht gezogen werden. In unserer Berliner Klinik haben wir ein spezielles Programm der totalen Gallenwegresektion durch Entfernung der gesamten Leber, des Lig. hepatoduodenale und des Pankreaskopfes mit gleichzeitiger Lebertransplantation begonnen; die Ergebnisse lassen zur Zeit noch keine endgültigen Schlüsse zu.

Büchler: Die Ergebnisse der Literatur zeigen, daß die Lebertransplantation bei Klatskin-Tumoren nicht zum Erfolg führt, weswegen eine Transplantation für diese Indikation nicht durchgeführt werden sollte.

Brambs: Eher nicht. Wenn eine Transplantation, dann wahrscheinlich eine En-bloc-Resektion von Leber, Duodenum und Pankreas.

\section{Weiterführende Literatur}

1 Barker EM, Winkler M: Permanent access hepaticojejunostomy. Br J Surg 1984;71:188-191.

2 Davids PHP, Groen AK, Rauws EAJ, et al: Randomized trial of self-xpanding metal stents versus polyethylene stents for distal malignant biliary obstruction. Lancet 1992;19:14881492.

3 Davids PHP, Tanka AKF, Rauws EAJ, et al: Benign biliary strictures. Surgery or endoscopy? Annu Surg 1993;217:237-243.

4 Jung M, Belletz M: Hepatobiliäres Stenting bei malignen und benignen Stenosen - welcher $\mathrm{Zu}$ gang ist der beste? Endosk Bildgebung 1995; suppl 2:47-52.

5 Knyrim K, Wagner HJ, Starck E, et al: Metalloder Kunststoffendoprothesen bei malignem Verschlußikterus. Ein randomisierter und pro- spektiver Vergleich. Dtsch Med Wochenschr 1992;117:847-853.

6 La Berge JM, Doherty M, Gordon RL, Ring EJ: Hilar malignancy: Treatment with an expandable metallic transhepatic biliary stent. Radiology 1990;177:793-797.

7 Moller Pedersen F: Endoscopic management of malignant biliary obstruction. Is stent size of 10 French gauge better than 7 French gauge? Scand J Gastroenterol 1993;28:185-189.

8 Pitt HA, Kaufman SL, Coleman J, et al: Benign postoperative biliary strictures. Ann Surg 1989;210:417-425.

9 Prat F, Chapar O, Ducot B, et al: Predictive factors for survival of patients with inoperable malignant distal biliary strictures: A practical management guideline. Gut 1998;42:76-80.
10 Shepherd HA, Royle G, Ross APR, et al: Endoscopic biliary endoprosthesis in the palliation of malignant obstruction of the distal common bile duct: A randomized trial. Br J Surg 1988; 75:1166-1168.

11 Smith AC, Dowsett JF, Russell RCG, et al: Randomized trial of endoscopic stenting versus surgical bypass in malignant low bile duct obstruction. Lancet 1994:344:1655-1660.

12 Speer AG, Russell FCG, Hatfield ARW, et al Randomized trial of endoscopic versus percutaneous stent insertion in malignant obstructive jaundice. Lancet 1987;7:57-62.

13 Trede M, Schwall G: Nutzen und Risiko präoperativer Gallendrainage aus chirurgischer Sicht. Dtsch Med Wochenschr 1985;110:556-559.

\section{Teilnehmer}

\section{Prof. Dr. H.-J. Brambs}

Abteilung für Röntgendiagnostik

Radiologische Universitätsklinik und Poliklinik

Universitätsklinikum Ulm

Steinhövelstraße 9

D-89075 Ulm

Prof. Dr. M. W. Büchler

Klinik für Abdominal- und Viszeralchirurgie

Inselspital Bern

CH-3010 Bern
Prof. Dr. M. Jung

Innere Abteilung

St. Hildegardis-Krankenhaus

Hildegardisstraße 2

D-55131 Mainz

Prof. Dr. P. Neuhaus

Transplantationszentrum

Virchow-Klinikum

Medizinische Fakultät der Humboldt-Universität

Augustenburger Platz 1

D-13353 Berlin
Prof. Dr. J. F. Riemann

Medizinische Klinik C

Klinikum der Stadt Ludwigshafen $\mathrm{gGmbH}$

Bremserstraße 79

D-67063 Ludwigshafen 